Ute Luig

\title{
Mobilitätsdiskurse in der Ethnologie
}

\section{Einleitung}

Die Ethnologie stand lange Jahre in dem Ruf, sich hauptsächlich mit ,Naturvölkern` zu beschäftigen, die als statisch und zeitlos galten. Als Wissenschaft von den (exotisch) Anderen grenzte sie sich durch dichotome Zuschreibungen, wie z.B. heiße und kalte Gesellschaften, eindeutig von modernen Gesellschaften ab, die für ihre Dynamik gesellschaftlicher Entwicklungsprozesse bekannt waren. Es erstaunt daher wenig, dass ein Begriff wie Mobilität in der ethnologischen Theoriebildung keine Rolle spielt. Dennoch zeigt sich bei näherem Nachdenken, dass verwandte Begriffe wie Wanderung, Expansion, Migration, Flucht, Reise in der Fachgeschichte sehr wohl wichtige Forschungsfelder darstellen, deren Bedeutung ohne Zweifel in den letzten Jahrzehnten beständig zugenommen hat.

Diese Behauptung möchte ich an drei verschiedenen Theoriekonstrukten, die mit sehr unterschiedlichen Fragestellungen einhergehen, kurz skizzieren. Dabei beziehe ich mich im Wesentlichen auf die drei Begriffe Wanderung, Expansion, Migration, die indes in der ethnologischen Tradition keine gesicherten und trennscharfen Begriffe darstellen, sondern eher aus der Perspektive der einzelnen Forscher definiert werden. Im Mittelpunkt meiner Darstellung steht der Begriff Migration, dessen Wandlung in den letzten hundert Jahren ich beschreibe. Ich beginne mit einem kurzen Hinweis auf die Bedeutung von Mobilität in der Debatte über Kulturkreise, die die deutschsprachige Ethnologie bis zu Beginn der I96oer Jahre beschäftigt hat, widme mich dann am Beispiel der Ausbreitung der Bantusprachen dem Begriff Expansion und beschreibe beispielhaft „imaginierte und reale Wanderungen“" für die Baule in der Elfenbeinküste und in Bezug auf die Tonga-Wanderarbeiter. Ein Resumée der heutigen Bedingungen von Migration beschließt meinen kurzen Überblick über dieses Thema.

\section{Diffusionismus und Mobilität}

Mobilität ist ein Begriff, der Veränderungen im Raum und in der Zeit beschreibt und im Kontext der Diffusionismus-Debatte Bedeutung erlangte. Die Vertreter des Diffusionismus beschäftigten sich mit der Frage, „ob ähnliche Objekte, die in weit voneinander ent-

I Dieses Begriffspaar übernehme ich der Beschreibung von Albert Wirz I997, 35 . 
fernten Gegenden existieren, einen gemeinsamen Ursprung haben, d.h. also von einem gemeinsamen Ursprungsort (Diffusionszentrum) ausgegangen sind und sich durch Wandel, Mission, Wanderungen, Nachahmung, direkte oder indirekte Entlehnung u.a. über die Welt verbreitet haben “. ${ }^{2}$ Einer der ersten Vertreter dieser Theorie war der Geograph und Völkerkundler Friedrich Ratzel, 3 der in Anknüpfung an den Münchner Zoologen Moritz Wagner eine Theorie der Kultur entwickelte, für die Raum, Zeit und Migration konstitutiv waren. In seiner Studie über afrikanische Bögen, Pfeile und Speere untersuchte er, in welcher Weise durch das Formkriterium historische Beziehungen offengelegt und kartographisch dargestellt werden konnten. „Er überwand die Raumscheu, welche bisher die Ethnographen gehindert hatte, mit weitausgreifenden Wanderungen der angeblich geschichtslosen Naturvölker und mit Kulturverbreitung über große Entfernung zu rechnen“.4 Die Überlegungen von Ratzel hinsichtlich der „Formverwandtschaften“ wurden von Bernhard Ankermann aufgegriffen, von Leo Frobenius im Begriff des Kulturkreises zusammengefasst und von Fritz Graebner,5 einem weiteren Museumswissenschaftler, systematisiert.

Es würde hier zu weit führen, die jahrzehntelangen Auseinandersetzungen über die Kulturkreise, die sich anfänglich lediglich auf Objekte der materiellen Kultur stützten, aber später auch Siedlungsformen, Bestattungsriten, Mythenmotive mit einschlossen, ${ }^{6}$ im Detail zu verfolgen. Sie waren ein Versuch, die Raumgebundenheit von Kultur ebenso wie ihre Veränderungen durch Formen der Mobilität zu reflektieren. Zwar kritisiert Mühlmann zu Recht den atomistischen Charakter dieses Kulturbegriffs, 7 weil Kulturen hier primär als Aggregate von Objekten und nicht als sinnhafte Strukturen verstanden wurden, doch generierte die jahrelange Auseinandersetzung über diesen Begriff wichtige Fragen der Anpassung und der inneren Dynamik von Gesellschaft.

\section{Die Bantu-Expansion}

Neben dem Sammeln und der Beschreibung von Objekten war die Analyse von Sprachen ein weiteres wichtiges Mittel zum Verständnis schriftloser Völker. Dieses in der Tradition der Aufklärung stehende Erkenntnisinteresse führte zu Beginn des I9. Jahrhunderts nicht nur zur Begründung der Indogermanistik, sondern seit der Mitte dieses Jahrhunderts auch zu einer intensiven Beschäftigung mit afrikanischen Sprachen, die vor allem von Missionaren untersucht wurden. Im Mittelpunkt standen die Bantusprachen, deren Erforschung

2 Hirschberg I998, 99.

3 Ratzel I89I.

4 Mühlmann I968, I25.

5 Graebner I967.

6 Vgl. dazu die Kritiken von Bormida I967 und Vansina I973.

7 Mühlmann I968, I28. 
mit dem Namen von William Bleek (I827-I875), einem in Bonn als Philologe ausgebildeten Bibelwissenschaftler, ${ }^{8}$ verbunden ist. Bleek hatte entdeckt, dass vom Atlantik bis zum Indischen Ozean und vom innertropischen Regenwald bis hinunter ins heutige Südafrika mit Ausnahme der Kalahari und großer Teile von Namibia - untereinander verwandte Sprachen gesprochen wurden. Er kreierte für diese Sprachgruppe, die je nach Klassifikation zwischen 400 und 600 Sprachen umfasst, das Wort bantu, das als Plural von muntu die Bezeichnung für Menschen ist. Er befasste sich ebenso wie Carl Meinhof (I857-I944) mit der Frage ihres Ursprungs und ihrer Ausbreitung.

Meinhof ging wie viele Wissenschaftler nach ihm von der Annahme aus, dass sich die Bantusprachen von einer gemeinsamen Ausgangssprache, dem so genannten Ur-Bantu, herleiteten. Er wendete zudem - ganz im Sinne der damaligen Zeit - diese rein linguistische Bezeichnung auch auf die Sprecher dieser Sprachen an, identifizierte also Sprache mit Ethnonym und folgerte daraus, dass die Ausbreitung der Sprache nur durch einen Wanderungsprozess erfolgt sein könne. Die Gleichsetzung von Sprache und Ethnos schätzten nicht nur Linguisten als methodisch hoch problematisch ein, sondern auch Historiker wie Thea Büttner und der Ethnologe Franz Boas, der auf unterschiedliche Entwicklungsdynamiken von Sprache und Kultur verwies. Trotz dieser grundsätzlichen Kritik, die auch von weiteren linguistischen Erkenntnissen gestützt wurde, war die Idee von den Bantu nicht mehr auszumerzen. Sie wurde - interessanterweise - auch von afrikanischen Intellektuellen aufrechterhalten, so u.a. von Théophile Obenga, einem kongolesischen Historiker und Schriftsteller, der eine gemeinsame Kultur und Weltsicht aller Bantusprecher postulierte. 9 Diese so genannte "philosophie bantoue", ro befeuerte jahrzehntelang die Diskussion darüber, ob es eine genuin afrikanische Philosophie gebe oder nicht. ${ }^{I I}$

\section{Diffusion und Differenzierung des Bantu}

Ebenso wie die Frage nach dem Urvolk beflügelte auch die Frage nach der Verbreitung der Sprache die Phantasie der mit dem Thema befassten Wissenschaftler. Ein halbes Jahrhundert nach Meinhof konnte Joseph $\mathrm{H}$. Greenberg zeigen, dass das Bantu mit einem Zweig der Benue-Cross River-Sprachen verwandt ist, die wiederum zu den Niger-Congo-Sprachen gehören. ${ }^{12}$ Er nahm daher an, dass das Ur-Bantu aus der Region Cross-River stammt. Das Zentrum lag seiner Meinung nach in Kamerun und in dem daran angrenzenden Teil von Nigeria. Jan Vansina zufolge wanderten Bantu-sprechende Gruppen von Nigeria aus nach

\footnotetext{
8 Vgl. Wirz 1997, 36.

9 Obenga 1985.

Io Vgl. Tempels i945.

II Vgl. Mudimbe ig88.

I2 Greenberg I963.
} 


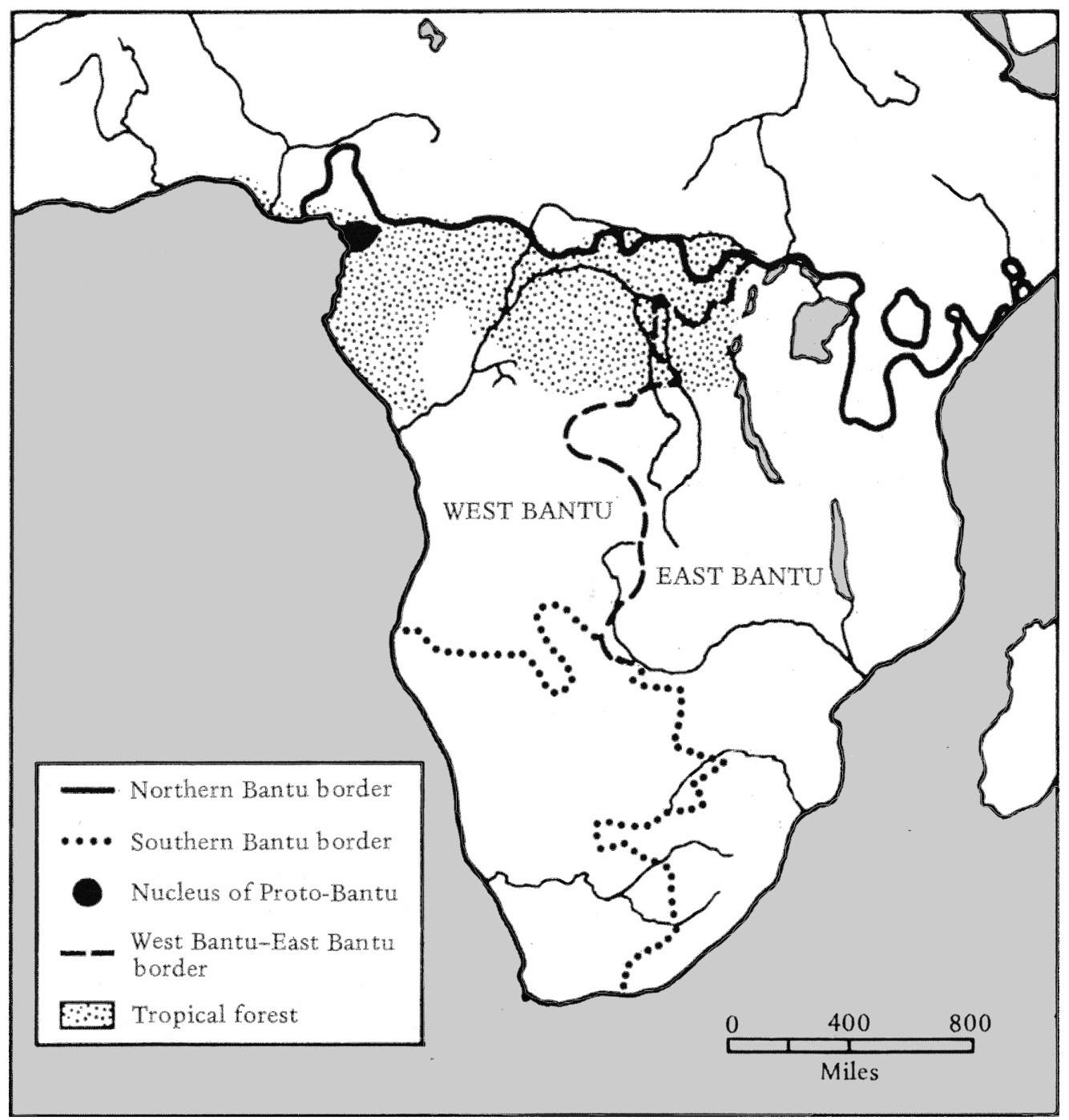

Abb. 1 | Die Verbreitung der Bantusprachen (aus Curtin et al. I978, 26)

Westen, Richtung Kamerun und Gabun. ${ }^{13}$ Von dort aus wandten sie sich entweder nach Norden entlang des Regenwaldes oder entlang seiner südlichen Ränder nach Zentral- und Südafrika (Abb. I).

Vansina gibt das Benue-Tal als Wiege der Bantusprachen an, die sich dort ungefähr 3000 v. Chr. in die westlichen und östlichen Bantusprachen teilten, die sich ihrerseits wei-

I3 Vansina I990, 49f. 
ter auffächerten. ${ }^{\mathrm{I} 4}$ Während sich die Ausbreitung der westlichen Bantusprachen eher langsam vollzog, diffundierten die östlichen und südlichen Bantusprachen vergleichsweise schnell, was sich aus der engen Verwandtschaft ihrer Sprachen ergibt. Das Jahr Iooo v. Chr. gilt hier allgemein als eine Zeit des kulturellen Umbruchs, ${ }^{15}$ für das südliche Afrika wird 800 v. Chr. als Richtschnur angegeben.

Neuere Untersuchungen, die z.T. auf unterschiedlichen Gliederungen der Bantusprachen basieren, ${ }^{\mathrm{T}}$ halten indes einen einheitlichen Ursprung der Bantusprachen (ähnlich dem Befund des Indogermanischen) nicht mehr für plausibel. So argumentiert Reinhard Klein-Arendt, dass „sicherlich alle Bantusprachen auf eine sprachliche Urquelle zurückgehen, die von Malcolm Guthrie als so genannte Proto-Bantu rekonstruiert wurde“, aber die Annahme einer linearen, von einer einzigen historischen Quelle ausgehenden Entwicklung sei unzureichend. Vielmehr müssen sich verschiedene, genealogisch verwandte Ursprachen zu unterschiedlichen Zeiten auf unterschiedlichen Wegen in das heutige Gebiet der Bantusprachen verbreitet und dort mit bereits vorhandenen verwandten sowie nichtverwandten Sprachen vermischt haben“. ${ }^{\text {I7 }}$ In diesem Prozess wird neuerdings auch dem Kuschitischen eine gestaltende Rolle für die ostafrikanischen Bantu zugeschrieben. ${ }^{18}$ Klein-Arendt konnte sich dabei auch auf die Ergebnisse des Kölner Linguisten Bernd Heine stützen, der nach einem systematischen Sprachvergleich von I37 Bantusprachen die These vertrat, dass sich ausgehend von dem von Greenberg postulierten Herkunftsgebiet „die Besiedlung der Südhälfte Afrikas durch die Bantu-sprachigen Völker in drei großen aufeinanderfolgenden Ausbreitungswellen vollzogen hat". ${ }^{\text {I9 }}$

\section{Was kennzeichnet die Proto-Bantu-Sprecher?}

Angesichts dieses recht komplexen Prozesses stellt sich die Frage, wer die Sprecher dieses Ur- oder Proto-Bantu waren, weshalb und auf welche Weise sie emigrierten?

Durch die Rekonstruktion des Ur- oder Proto-Bantu, die vor allem im epochalen Werk von Malcom Guthrie „Comparative Bantu“ festgeschrieben ist, ${ }^{20}$ gelang es mit Hilfe von Wortfeldanalysen Aufschluss über ihre Wirtschaft, die soziale Ordnung und über die Religion zu erhalten. ${ }^{2 \mathrm{I}}$ So stellten die Sprecher des Proto-Bantu in Kamerun und den benachbarten Gebieten Keramik her, hatten aber noch keine Kenntnisse der Eisenverhüttung. ${ }^{22}$ Es

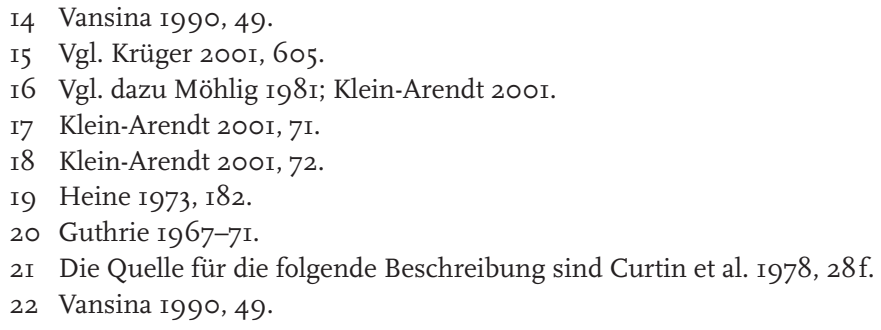


handelte sich, wie Vansina schreibt, um eine neolithische Kultur, die je nach Region auf Fischfang, Jagd und dem Anbau von Feldfrüchten basierte. Jene Gruppen, die am Rande des Regenwaldes siedelten, bauten Yams und Ölpalmen an, die in der Savanne lebenden Gruppen kultivierten Hirse und andere Getreidesorten. Auch hielten sie Kleintiere, wie Schafe und Ziegen. Verwandtschaftsstrukturen sowie Polygynie bildeten die Grundlage der sozialen Beziehungen, während Worte für Führerschaft (leader) auf eine hierarchische politische Organisation hindeuteten, die sich offensichtlich auf territoriale wie auf religiöse Bereiche (Eastern Bantu) erstreckte.

Motive und Formen der ,Migration‘

Während sich die Hauptrichtungen der ,Bantu-Migrationen' anhand linguistischer Analysen zumindest ansatzweise bestimmen lassen, wissen wir über das Wie und Warum dieser Prozesse noch immer sehr wenig.

Im Einklang mit den Vorstellungen von den Bantu als einheitlichem Volk nahmen Carl Meinhof wie auch der englische Kolonialpolitiker Sir Harry Johnston (I858-I927) an, dass sich die Sprache „im Zusammenhang mit einem gewalttätigen Wanderungsprozeß“ verbreitete; Johnston sprach gar von „Horden gewalttätiger Invasoren“.23

In diesen Bildern wurden - ähnlich wie in der Kulturkreisdebatte - Bilder einer Völkerwanderung evoziert, die Vansina wie folgt beschreibt:

The image is generally that one day thousands started to move, destroying everything in their passage, or fleeing some conqueror, and that the emigrants walked in a beeline from their point of ,origin' to the point where they lived at the end of the Igth century, that people forced other people out and threw them on the road. ${ }^{24}$

Obwohl solche Massenmigrationen in der afrikanischen Geschichte Vansina zufolge nur in wenigen Fällen belegt sind, u. a. für die Jago in Zaire, die Ngoni und Kololo im südlichen Afrika, haben die Vorstellungen darüber die Imaginationen der Historiker beflügelt. Allerdings sprechen aus praktischer Sicht sehr gewichtige Gründe gegen eine solche Massenwanderung:

Die zu durchquerenden Gebiete waren ökologisch höchst verschieden und erforderten zum Überleben genaue Kenntnisse von Flora und Fauna. Die zentralafrikanischen Regenwälder gehören zu den prekärsten Ökosystemen der Welt, da ihr Boden nur über eine geringe Humusschicht und geringe Nährstoffe verfügt. Der Boden dient daher hauptsächlich als Stütze im Wachstumsprozess der Pflanzen, die mehrere Höhenstockwerke bilden

23 Wirz I997, 37.

24 Vansina I966, I7. 
und dadurch ein eigenes Mikroklima erzeugen, das wegen der hohen Luftfeuchtigkeit die Austrocknung des Bodens verhindert. Trotz dieser günstigen Regenerationsbedingungen bietet der Regenwald aber nur begrenzte Möglichkeiten für Agrikultur, die wegen der Bodenqualität keine größeren Menschengruppen langfristig ernähren kann. Die zahlreichen Flüsse, Seen und Sümpfe stellen zudem schwierig zu überwindende Barrieren dar, die das Vorwärtskommen großer Gruppen von Personen behindern. Wie schwierig, gefahr- und qualvoll diese Art des Fortkommens für europäische Eroberer war, beschreibt Johannes Fabian in seinem Buch „Wissenschaft und Wahn in der Erforschung Zentralafrikas“. ${ }^{25}$ Diese Form des Wissens war, wenn sie denn überhaupt existierte, unter den Führungspersonen oder Spezialisten, wie Jäger und Heiler, in einer Ethnie verbreitet, da es sich um sehr spezifische Kenntnisse handelte.

Die vorhandene Evidenz bestärkt deshalb für die ,Bantu-Migration' die Annahme, dass es sich vor allem um Kleingruppen handelte, die entweder auf Lineage- oder Clanebene organisiert waren und sich nur langsam fortbewegten. Nach Vansinas Berechnungen legten die Migranten vom Sanaga-Fluss bis zum Congo 22 km in Io Jahren zurück; andere brauchten 470 Jahre um $400 \mathrm{~km}$ zu überwinden, was einem Zehnjahresdurchschnitt von $\mathrm{I} 7 \mathrm{~km}$ entspricht. ${ }^{26}$ Vansina bezeichnete diese Form der Wanderung als drift, eine Bezeichnung, für die es im Deutschen in diesem Kontext keine wirklich adäquate Übersetzung gibt. Territoriale Expansion scheint mir für diese allmähliche Aneignung des Raums noch am naheliegendsten. Im Unterschied zur Migration, die von Punkt A nach Punkt B verläuft, impliziert Expansion eher eine Ausbreitung in der Fläche, die größere Zeitspannen einschließt.

Migration, Wissen, Herrschaft

Ebenso wie die Annahmen über den Ablauf der Expansion divergierten, wurden auch verschiedene Erklärungen für ihre Ursachen gegeben. Entsprechend der für Migrationstheorien typischen „push and pull“-Faktoren wurde von Roland Oliver eine Bevölkerungsexplosion $^{27}$ als Folge verbesserter Agrartechniken angenommen, die durch Kenntnisse in der Eisenverarbeitung unterstützt wurden. ${ }^{28}$ Vansina hingegen nannte als Motive für die Migration ökonomische Entwicklungen, wie die Urbarmachung neuen Landes, das Erschließen neuer Jagdgründe oder besserer Siedlungsbedingungen. ${ }^{29}$ Er schloss Bevölkerungsdruck für die Gebiete in Kamerun und Kongo ebenso aus wie die Eisenverhüttung, da Wörter für Eisen erst nach der Ausdifferenzierung der Bantusprachen im Vokabular ein-

25 Fabian 200 I.

26 Vansina 1990,55 .

27 Oliver I966; vgl. auch Heine et al. I977, 63.

28 Ähnlich Wrigley i960.

29 Vansina I990, 55. 
zelner Gruppen auftauchten und dadurch zweifelsfrei einer späteren Epoche angehörten. Interessanterweise werden auch ökologische Gründe als Ursache für Migration genannt. So konnte im Jahre 2004 eine Forschergruppe aus Frankfurt unter der Leitung von Breunig nachweisen, dass aufgrund von Pflanzenfunden, deren Anbau man bisher ausschließlich mit der Savanne assoziiert hatte, eine Veränderung des Klimas im zairischen Regenwald stattgefunden haben muss. Der Anbau dieser Pflanzen sei durch die Schrumpfung des Regenwaldes ermöglicht worden, dessen Folge klimatische Veränderungen gewesen seien. ${ }^{\circ}$

Aufgrund dieser Entdeckung ist mit grundlegenden Konsequenzen für die Besiedlungsgeschichte des Regenwaldes zu rechnen. Während nämlich bis in die I980er Jahre die Pygmäen als autochthone Bevölkerung des Regenwaldes gegolten haben, gerät diese Annahme nun grundlegend ins Wanken. Sie wurde allerdings auch schon durch die Forschungen von Terese B. Hart und John A. Hart zumindest für Teile des zairischen Regenwaldes infrage gestellt, ${ }^{\text {I }}$ da das Ehepaar nachweisen konnte, dass die Pygmäen aufgrund eines saisonalen Ressourcenmangels auf die Unterstützung lokaler Feldbauern angewiesen sind. Zwar hatte auch Colin M. Turnbull schon die enge ökonomische Kooperation zwischen Bauern und Wildbeutern beschrieben, doch hatte er keinerlei Zweifel, dass die Pygmäen nicht die ersten Siedler des Regenwaldes seien.32 Dass sich in dieser Frage noch weitere neue Erkenntnisse ergeben können, ist keineswegs ausgeschlossen.

Zusammenfassend lässt sich festhalten, dass die Durchquerung und Aneignung von Raum im Verlauf der Bantu-Expansion an ein spezifisches Wissen gekoppelt war, das genaue Kenntnisse über die Umwelt voraussetzte. Diese konnten nur in langen Zeitfenstern erworben und umgesetzt werden. Der Erfolg der Expansion basierte aber auch auf einer Verfügung über technologisches Wissen, das mit Kenntnissen der Eisenverarbeitung gleichgesetzt wurde. Diese (in der Einschätzung kolonialer Autoren) höher entwickelte Technik wurde in ihren Überlegungen mit waffentechnischer Überlegenheit verbunden. Die - wie oben gezeigt - immer wieder anklingenden Aussagen über die Überlegenheit der Bantu basieren auf diesem Wissensvorsprung, der ihnen die Ausübung von Macht ermöglichte.

Hier klingt ein Topos an, der für die vorkoloniale Geschichte Afrikas von großer Bedeutung ist, da Migration und Herrschaft von den meisten Autoren zusammengedacht werden. Anders als im Diskurs des 2I. Jahrhunderts, in welchem die Migranten als Herrschaftsunterworfene beschrieben werden, gelingt es diesen Theorien zufolge den einwandernden Gruppen, sich als Herren zu etablieren und die autochthone Bevölkerung zu ,überlagern‘. Im Diskurs der Überlagerung wird Herrschaft immer exogen begründet und auf militärische Effizienz, straffe soziale Organisation, ökonomische Entwicklung und/ oder überlegene Rassenzugehörigkeit zurückgeführt.

30 Vgl. dazu Eggert i992.

3I Hart/Hart i986.

32 Turnbull i983. 
Symbolhaft für das Letztere steht die Hamitentheorie, die u.a. von Charles G. Seligman 33 und Carl Meinhof 34 vertreten wurde. Die Hamiten, unter die die Hirtenvölker des Sudans, Ost- und Südafrikas aber auch die Fulbe Westafrikas subsumiert wurden, galten als die eigentlichen Kulturträger in Afrika. Diesen ,hellhäutigen' Hirtenvölkern, die aus dem Mittelmeerraum, respektive Ägypten oder Irak nach Afrika eingewandert sind, wurden alle großen Kulturleistungen (Megalithkulturen in Uganda und Zimbabwe) sowie die Errichtung der Zwischenseen-Königreiche zugeschrieben. Die Hamitentheorie bestätigte daher nochmals - aus scheinbar berufenem Munde - dass ,Negroide‘ keine großen Kulturleistungen hervorgebracht haben und das Wissen sowie der technologische Fortschritt etwas genuin ,Weißes' sei.

Welches Gefahrenpotential allerdings in dieser rassistischen Überhöhung des Hirtentums liegt, zeigt die neuere Geschichte Ruandas. Obwohl Hutu und Tutsi beide Bantusprecher sind, akzeptierte die belgische Kolonialregierung die politische Überlegenheit der Tutsi als letztlich ethnisch begründet35 und vertiefte somit die wachsende Kluft zwischen den beiden Gruppen, die den Hutu dann zur Legitimation für den Genozid an den Tutsi diente. ${ }^{36}$ Gerade an diesem tragischen Ereignis lässt sich ablesen, dass die Bantu-Expansion, deren interdisziplinäre Forschungsgeschichte inzwischen auf eine mehr als hundertjährige Forschungstradition zurückblicken kann, Teil einer zu rekonstruierenden Vergangenheit ist, die zum Verständnis der Gegenwart ebenso wichtig ist wie für die Gestaltung der Zukunft. Sie wurde in der postkolonialen Ära zum Verhandlungsfeld afrikanischer Identität, die zwar koloniale Rassismen beklagte, sich aber auch nicht immer gänzlich von ihnen - etwa im Hinblick auf die Hamitentheorie - freimachen konnte. So ist die Feststellung von Bethwell A. Ogot, "(that) state formation can only be explained in terms of environmental, political and economic factors, and not in terms of race or ethnic groups",37 eine notwendige Warnung in einer Situation, in der Geschichte zum Narrativ des Ethno-Nationalismus umfunktioniert zu werden droht.

\section{Imaginierte und realisierte Wanderungen der Baule}

Wandersagen als Selbstbeschreibung

Die Suche nach der Identität der afrikanischen Völker wurde von der kritischen Afrikawissenschaft im Zeitalter des Postkolonialismus als Herausforderung verstanden. Im Unterschied zu den Forschungsbedingungen der kulturhistorischen Schule hatte sich die Quel-

33 Seligman I930.

34 Meinhof 1938.

35 Vgl. Neubert/Brandstetter I996.

36 Brandstetter 2005 .

37 Ogot I968, I30. 
lenlage enorm verbessert, da neben archäologischen Befunden, linguistischen Analysen und oralen Traditionen nun auch eine Vielfalt von Schriftquellen existierte. Zwar waren die frühen Texte - abgesehen von einigen Ausnahmen lokaler Intellektueller - von ehemaligen Kolonialbeamten verfasst worden und unterlagen daher einem eindeutigen kolonialen Bias, doch hatten die Interessierteren unter ihnen, wie etwa Delafosse in der Elfenbeinküste oder Bowdich in Ghana, eigene empirische Untersuchungen vor Ort durchgeführt und sich um die Aufnahme oraler Traditionen, Mythen und Legenden verdient gemacht. In vielen dieser oralen Traditionen spielen Wanderungen eine zentrale Rolle, um den Ursprung und die Herkunft eines Volkes zu erklären. Dieser Begriff der Wanderung kommt den Vorstellungen, die in der ,Bantu-Expansion“ damit verbunden wurden, nämlich die Erschließung von Land und die Begründung von Herrschaft, erstaunlich nahe. Aufgrund der von Vansina angewandten historisch-kritischen Methode der Textanalyse lassen sich die in den Mythen und Sagen enthaltenen Beschreibungen nicht mehr als Widerspiegelung von Realität lesen, ${ }^{8}$ sondern als zeitgeschichtliche Dokumente, die einer kontextuellen Interpretation bedürfen. Besondere Bedeutung fällt in diesem Interpretationsprozess den verschiedenen Varianten eines Textes $\mathrm{zu}$, da diese meist dissidente Sichtweisen wiedergeben. Neben der zeitlichen Einordnung bedarf es jedoch auch einer Dekodierung der im Text verwendeten Symbole und Metaphern,39 die Aufschluss über gesellschaftliche Organisationsstrukturen, kulturelle und moralische Wertvorstellungen sowie das Verhältnis der Menschen zur Natur und zu den Göttern geben können. Wie groß die Differenz zwischen einem Mythos und der ,Realgeschichte‘ sein kann, möchte ich etwas verkürzt an der Sage von Aura Pokou erläutern, die I9०o von dem französischen Kolonialhistoriker Maurice Delafosse aufgezeichnet wurde. $4^{\circ}$

Delafosse zufolge gelang es der Königin Aura Pokou auf der Flucht vor den Häschern des Ashanti-Königs Opoku Ware, mit einigen Getreuen den Comoé-Fluß zu überqueren, nachdem sie auf Bitten der Flussgeister ihren eigenen Sohn geopfert hatte. Die Flüchtlinge waren jedoch auf dem anderen Ufer zunächst nicht willkommen und mussten sich durch militärische Auseinandersetzungen ihren Weg bahnen. Dabei unterwarfen sie acht verschiedene Gruppen, die in unterschiedlichen Funktionen in das von Aura Pokou im Zentrum der Elfenbeinküste gegründete Königreich integriert wurden.

In dieser ätiologischen Sage, die alle Merkmale einer Wandersage enthält,4 ${ }^{\mathrm{I}}$ finden sich alle klassischen Themen wie Flucht, Migration, Unterwerfung, Widerstand und die Errichtung zentralisierter Herrschaft (Königreich). Die Analyse der verschiedenen Varianten ermöglichte jedoch einen komplexeren Einblick in die Ethnogenese der Baule, da ich durch einen historisch-kritischen Vergleich dieser Varianten aufzeigen konnte, dass so-

38 Vansina I973; ders. I985.

39 Vgl. dazu Luig I984, I78-I94.

40 Delafosse I900; vgl. dazu Luig 1985.

4I Vgl. dazu Vansina 1974. 
wohl die Massenmigration, die anschließende Eroberung der Elfenbeinküste als auch die Gründung des Königreichs keineswegs in allen Fassungen der Sage tradiert werden, sondern vornehmlich in dem von Delafosse publizierten Text. In den Varianten der ,Dissidenten' fehlen diese Hinweise völlig oder sie werden durch eine entsprechende Anordnung der Texteile zu normativen Aussagen über politische Bedingungen von Herrschaft verdichtet, ohne die faktische Herrschaft von Aura Pokou zu bestätigen. Der von Delafosse publizierte Text war ihm vom damaligen ,König' der Warebo diktiert worden und diente angesichts der kolonialen Situation der Legitimation des Führungsanspruchs der Baule über andere ethnische Gruppen in der Elfenbeinküste; ein Ansinnen, das durch die Übersetzung ins Französische durch Delafosse und die schnelle Ausbreitung der Sage sein Ziel nicht verfehlte. Delafosse, wie viele Historiker nach ihm, hatte noch nicht erkennen können, dass orale Traditionen kulturelle Archive sind, die in symbolisch verschlüsselter Form das Selbstverständnis eines Volkes verkörpern, das indes nur nach kritischer Überprüfung durch andere Quellen als ein Element in der Rekonstruktion der faktischen Geschichte $\mathrm{zu}$ verwenden ist. Die Sage von Aura Pokou wurde daher bis in die I970er Jahre in allen wichtigen Geschichtsbüchern in der Delafosschen Interpretation tradiert, bis der englische Historiker Timothy Weiskel durch ein intensives Quellenstudium französischer Kolonialquellen ihre grundlegenden Annahmen falsifizieren konnte. Weiskel widerlegte die Massenmigration und beschrieb die Phase der ,Eroberung' als kulturelle Assimilation durch Einheirat und eine allmähliche demographische Expansion. ${ }^{42}$

Anknüpfend an diese Untersuchungen gelang es mir durch Forschungen bei den Randgruppen des so genannten ,Baule-Königreichs', also den in der Sage aufgeführten Dissidenten, deren Version der politischen Organisationsstruktur und vor allem ihre lokalen Wanderungsgeschichten aufzunehmen. Obwohl auch in meinen Interviews offiziell die Sage von Aura Pokou immer wieder erzählt wurde, vermittelte die Rekonstruktion der Familien- und Lineage-Geschichte ein viel differenzierteres Bild. In ihm lassen sich zwar ebenfalls Hierarchisierungsprozesse erkennen, doch werden diese infolge der segmentären Dynamik immer wieder nivelliert.43

\section{Die Expansion der Baule (1720-1900)}

Die Baule leben im Zentrum der Elfenbeinküste, im so genannten Baule V (Abb. 2), das bis weit nach Süden reicht und ca. $30000 \mathrm{~km}^{2}$ umfasst. Sie sind die größte ethnische Gruppe der Elfenbeinküste, die ihrerseits wiederum aus 28 verschiedenen Untergruppen besteht, und in einflussreicher Weise die politische und ökonomische Entwicklung des Landes geprägt hat.

42 Weiskel i980.

43 Vgl. Sigrist i968. 


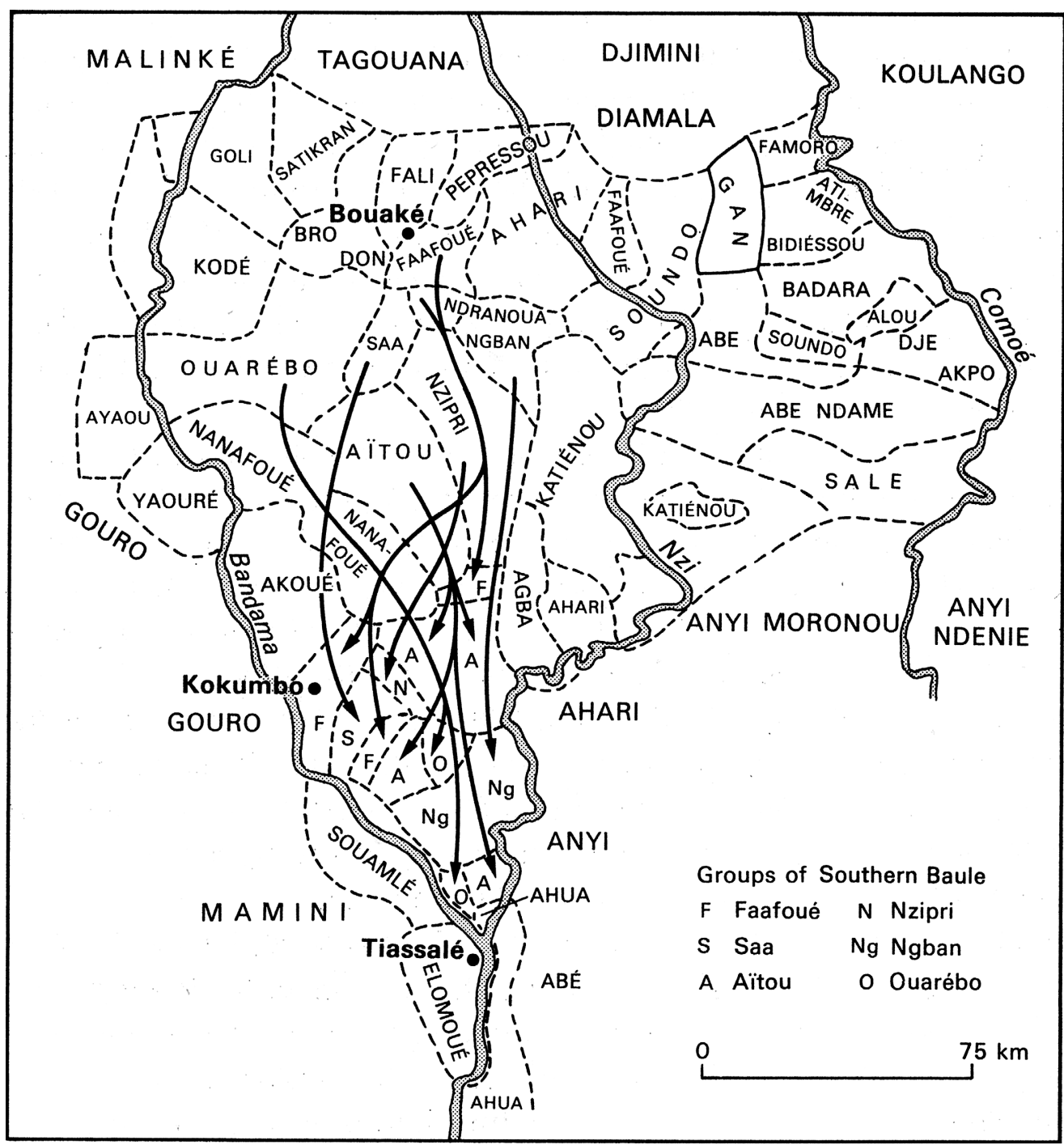

Abb. 2 | Die Haupt-Baule-Gruppen und ihre südliche Migration im I9. Jahrhundert (aus Weiskel I980, I2)

Meine Interviews, die ich vornehmlich mit Lineage-, Clan- und Dorfchefs führte, belegten, dass entgegen des verbreiteten Stereotyps der Massenmigration nur einzelne Familienkerne ,wanderten' und ihre Verwandten erst nachholten, wenn sie eine entsprechende Infrastruktur etabliert hatten. Die Gründung eines neuen Dorfes erforderte detaillierte ökologische, religiöse und soziale Kenntnisse. Dazu gehörten die Vertrautheit mit den geographischen Gegebenheiten, das Abschätzen von Gefahren, etwa bei der Überquerung von Flüssen und Sümpfen ebenso wie Kenntnisse der lokalen Flora und Fauna. Der Rhyth- 
mus der Expansion wurde in entscheidender Weise durch die Strukturen des Raumes geprägt, dem differenzierte Vorstellungen über das Verhältnis von Natur und Kultur zugrunde lagen.

Natur und Kultur als distinkte europäische Kategorien der Gliederung des Raums sind keine universalen Kategorien. In afrikanischen Sprachen existiert z. B. kein Begriff für Natur. Stattdessen unterscheiden die Baule zwischen der von Menschen bewohnten Welt, dem (Dorf - kro), und der durch Menschen transformierten Welt. Dazu gehören die Wege (ati), die aus dem Dorf herausführen, das Feld (fie), das Campement (niamonoué) und der Dorfrand (dassie). Die Wildnis gehört zu einer dritten Kategorie, der unbewohnten Welt, die im Fall der Baule mit dem Wald identisch ist; in anderen Gesellschaften aber auch durch den Busch oder die Stadt repräsentiert wird.44 Auch der Wald ist in sich wiederum differenziert, da er sowohl aus heiligen Hainen besteht als auch aus Heterotopien, Plätzen, in denen Abfall oder gefährliche Materie, wie z.B. die Leichen von Selbstmördern oder Hexen gelagert werden, die aus der menschlichen Gesellschaft ausgestoßen wurden. Je weiter entfernt ein Territorium vom Dorf ist, umso gefährlicher wird es für die Menschen. Diese Gefahr wird vor allem den Geistern zugeschrieben, die Feld und Wildnis bevölkern und eine Gegenwelt zur Welt der Menschen bilden. Die Wildnis ist ein Symbol für das Nicht-Domestizierte, Nicht-Kontrollierbare, Chaotische. Sie ist aber auch ein Synonym für Fruchtbarkeit sowie für Bedrohung und Gefahr.45 In diesen polysemischen Bedeutungen drücken sich die Widersprüchlichkeit und Ambivalenzen aus, mit der die Baule der Wildnis gegenüber stehen.

Sich diese Wildnis anzueignen, sie urbar zu machen, bedurfte gewisser Qualifikationen, die in einer Art Sozialisationsprozess erworben werden mussten. Wie meine eigenen Daten für die Elfenbeinküste und die von Carola Lentz für Ghana ${ }^{4}{ }^{6}$ zeigen, war es zunächst nur Jägern, kriegserprobten Anführern oder auch Propheten erlaubt, die Grenze zur Wildnis zu überschreiten und das Anlegen neuer Siedlungen zu planen. Bei ihnen handelte es sich um Personen, die überdurchschnittliches ökologisches Wissen hatten und enge Beziehungen zu den Ahnen und Geistern unterhielten, die gewöhnlichen Menschen nicht zugänglich waren. Nur sie verfügten daher über die religiöse Autorität für ein solches Unterfangen, das einen Pakt mit den Geistern der Erde und des Waldes etc. voraussetzte, der durch entsprechende Opfer besiegelt werden musste. Erst wenn dieser Pakt erfolgreich abgeschlossen war, konnten einzelne, meist jüngere Familienmitglieder es wagen, neue Felder anzulegen und auf ihnen temporäre Behausungen zu bauen, die als niamonoué (campement) bezeichnet werden. Die Bewohner dieser Siedlungen hatten nur begrenzte Rechte, da z.B. Geschlechtsverkehr, Rechtsstreitigkeiten sowie alle Rituale nur in ihrem Herkunftsdorf vollzogen werden konnten.

44 Vgl. Klute I995.

45 Vgl. dazu Luig/von Oppen I995, 6 f.

46 Lentz 2006. 
Diese Formen der Landnahme, die konkret einer Segmentation gleichkam, waren innerhalb der Verwandtschaftsgruppen genauestens geregelt, da sie ein Mittel zu einer kontrollierten territorialen Expansion darstellten. Die Neugründung solcher niamonoué erfolgte nur wenige Kilometer, in der Regel nicht mehr als ein bis zwei Tagesmärsche von der alten Siedlung entfernt. Sie gruppierten sich häufig radial um ihre ehemaligen ,Ursprungsdörfer', von denen sie durchschnittlich fünf bis sieben Jahre abhängig blieben, bevor sie einen vollen Rechtsstatus als Dorf erlangten. Erst wenn die Bewohner die ihnen auferlegten Pflichten absolviert hatten und sich die neue Gründung als lebensfähig erwies, erhielten sie ihrerseits den Status eines Dorfes. Dieser wurde durch die Vergabe eines Stuhls ausgedrückt, der ein Symbol politischer Unabhängigkeit darstellte, weil er eine neue Ahnenlinie begründete. Dem Inhaber eines solchen Stuhls wurden damit alle Rechte (Landrechte und rituelle Autorität über ein Territorium) verliehen, die mit einer solchen Erstbesiedlung verbunden waren und durch das „religiöse“ Amt des Erdherrn (assie kpengben) ausgedrückt wurden.

In der den Baule eigenen Siedlungsstruktur zwischen Dorf - campement(s) - Dorf drückte sich die soziale Dynamik zwischen Segmentation, Integration und Akkumulation von Menschen schon in der Anlage des Dorfes aus. Die Lebensfähigkeit solcher Neugründungen, denen jeweils ein Oberhaupt vorstand, das je nach Größe der Siedlung entweder durch einen aulokpengben (Gehöftchef) oder einen akpaswakpengben (Lineagechef) vertreten wurde, war nicht allein durch die Reproduktion der eigenen Gruppe zu gewährleisten, sondern bedurfte auch der Integration von Gruppenfremden, die sich auf vielfältige Weise vollzog. Häufig geschah dies durch die Einheirat von Händlern, die auf der Suche nach Allianzbeziehungen und HandelspartnerInnen waren. Die Einheirat in eine ortsansässige Familie ermöglichte ihnen den Aufbau von Relaisstationen, die zu wichtigen Knotenpunkten in den Fernhandelsnetzen zwischen Wald und Savannen wurden. Um eine größtmögliche Integration von Personen zu ermöglichen, veränderten die Baule ihr Verwandtschaftssystem von einem matrilinearen in ein kognatisches47 System mit matrilinearer Akzentuierung. Ferner spielten sowohl Adoptionen von Kindern aus verwandten Familien (fosterage) als auch von (ausgestoßenen) Einzelpersonen oder zugewanderten Familien eine große Rolle, $4^{8}$ die zur inneren Differenzierung der Hauseinheiten (aulo) wie der Dörfer beitrugen. Die sich daraus ergebenden Strukturen sozialer Abhängigkeiten verstärkten sich noch durch die Integration von Haus- und Schuldsklaven im I9. Jahrhundert, die am

47 In einem kognatischen System kann ein Individuum seine Beziehung zu einem Ahnen entweder über die mütterliche oder väterliche Linie herleiten (ambilinear).

48 Seit den I950er Jahren wanderten auch so genannte Six mois, Kontraktarbeiter aus Burkina Faso und Mali in die Elfenbeinküste und verdingten sich auf den Kaffeeplantagen der Baule. Vielen gelang es, von ihren ehemaligen Arbeitgebern Land zu pachten. So entstanden in den Baule-Dörfern noch so genannte Mossi-Quartiere, deren Bewohner islamischen Glaubens waren. Sie interagierten nur in sozialen und politischen Belangen mit ihren Baule-Nachbarn, hielten sich ansonsten weitgehend für sich und begründeten Siedlungsgemeinschaften, die wir heute als Parallelgesellschaften bezeichnen. 
Rand des Regenwaldes entweder gegen lokal gefördertes Gold eingetauscht werden konnten oder in Zeiten der Krise sogar nur gegen Lebensmittel.

Die äußerst erfolgreiche Siedlungsgeschichte der Baule war sowohl verbunden mit ökonomischen Anreizen - so spielte die Suche nach Gold und fruchtbaren Böden die Hauptrolle - als auch mit dem Aushandeln von Konflikten. Wie in vielen afrikanischen Gesellschaften bildeten Hexereianklagen und Führungsstreitigkeiten wichtige Ursachen für Abwanderung und die Neugründungen von campements. Diese Konflikte waren jedoch gesamtgesellschaftlich betrachtet nicht wirklich destruktiv, da sie aufgrund der segmentären Dynamik, die Beziehungen der Abhängigkeit regelte, gesamtgesellschaftlich integrativ wirkten. So wechselten differenzierte Strukturen gradueller sozialer Ungleichheit, die in sich transformierbar waren - wie etwa zwischen Alten und Jungen, Männern und Frauen,49 Einheimischen und Fremden - mit ausgeprägteren hierarchischen Beziehungen zwischen Einheimischen, Schuldsklaven und Sklaven ab. Was zählte, war nicht so sehr die Macht über Land, das bis in die Mitte des 20. Jahrhunderts ausreichend vorhanden war, sondern über Gold und Menschen. Die Austauschmöglichkeit von Gold gegen Menschen bildete somit den Motor der Expansion, da sie immer wieder neue Optionen der Integration eröffnete. Dass sich aus solchen Strukturen tatsächlich Königreiche oder ganze Imperien unter günstigen ökonomischen und militärischen Bedingungen entwickeln konnten, zeigt das Beispiel des benachbarten Ashanti-Königreichs. $5^{\circ}$

\section{Migration, Identität, Diaspora}

Diese historische Darstellung früherer Migrationsprozesse sollte jedoch nicht den Eindruck vermitteln, dass sich die Ethnologie nicht mit ,modernen‘ Formen der Migration seit dem 20. Jahrhundert beschäftigt hat. Wie Andreas Ackermann betont, gibt es zwar keine Ethnologie der Migration, ${ }^{51}$ aber seit den Arbeiten der Chicago-Schule in den I92oer Jahren hat eine differenzierte Auseinandersetzung mit verschiedenen Facetten von Migration stattgefunden. Aus dieser weitgefächerten Palette, in der einerseits zwischen freiwilliger und unfreiwilliger Migration (= Flucht) unterschieden wird, und innerhalb der freiwilligen Migration dann nochmals nach Gründen (Hunger, Armut, Bildungs- und Jobsuche etc.) differenziert wird, möchte ich mich hier lediglich mit der Suche nach Arbeit beschäftigen.

Die Analyse der so genannten Wanderarbeit wurde zu einem Markenzeichen der Manchester-Schule, die von Max Gluckman in den I950er Jahren begründet wurde. Als Direktor eines der wichtigsten Forschungsinstitute in Schwarzafrika, dem ehemaligen

49 Die Macht der Frauen war primär abhängig von der Zahl ihrer Kinder, von ihrer Verfügung über Gold sowie Sklaven und nicht geschlechtlich definiert.

50 Vgl. Luig I980.

5I Ackermann I997, I. 
Rhodes Livingstone Institut in Lusaka/Sambia beschäftigten sich Gluckman und seine Schüler (Turner, Epstein, Mitchell u.a.) schon seit der Mitte der I940er Jahre mit den Arbeitsverhältnissen im Copperbelt im Norden Zambias. ${ }^{22}$ Dessen Arbeiterschaft rekrutierte sich ebenso wie diejenige in den Minen Südafrikas aus Wanderarbeitern, die aus den umliegenden Ethnien stammten. Sie wurden anfänglich als Kontraktarbeiter beschäftigt, die höchstens über eine sechsmonatige bis zweijährige Arbeitserlaubnis verfügten. Eingestellt unter ausbeuterischen Arbeitsbedingungen und mit minderen Rechten im Vergleich zu den weißen Arbeitern in Bezug auf Lebens- und Wohnverhältnisse (vor allem in Südafrika) stellten sie eine mobile Arbeitskraftreserve dar, die jederzeit kündbar war. Der Begriff Wanderarbeiter bezog sich deshalb auf ihr kontinuierliches Pendeln zwischen Land und Stadt, das sowohl eine Folge sozialer und ökonomischer Zwänge als auch eine Reaktion auf die Verheißungen der Stadt jenseits dörflicher Normenkontrolle war. In den Gesellschaften des südlichen Afrika transformierte sich die Teilnahme an der Wanderarbeit in einen Initiationsritus für Männlichkeit53 mit einem hohen Prestigefaktor. Für diese erste Forschergeneration ergaben sich daher ganz neue Fragen, die sich vor allem mit Problemen der Integration dieser vom Land stammenden Arbeiter in eine schon damals komplexe Industriegesellschaft verbanden. Robert Redfield54 hatte in seinen Studien über Yucatan die folk society (Land), die er als „klein, isoliert, nicht-schriftkundig, homogen und mit einem starken Gefühl der Gruppensolidarität ausgestattet" beschrieb, grundlegend vom „urban way of life"55 unterschieden, der die gegenteiligen Werte verkörperte. In Redfields Konzeption verkörperten diese beiden Lebensweisen zwei Welten, die er als "folk-urban continuum " bezeichnete. ${ }^{6}$ In einer kritischen Auseinandersetzung mit dieser Zwei-Welten-Theorie, die die Migration in die Stadt mit Problemen der „Detribalisierung“ verband, vertrat Gluckman daher die inzwischen berühmte These: „An African townsman is a townsman, an African miner is a miner“. 57 Diese Situationsanalyse einer kontextbezogenen Identität ging davon aus, dass sich die Wanderarbeiter jeweils ihrem kulturellen Milieu anpassten und entsprechend dessen Normen handelten. Diese These, die schnell Verbreitung fand, sollte durch die Untersuchungen der Netzwerke von Wanderarbeitern untermauert werden. Ausgehend von der Arbeit von Elizabeth Bott $5^{8}$ interessierten sich die Forscher für die Verteilung von StadtLand-Beziehungen ihrer Informanten ebenso wie sie der Frage nachgingen, inwieweit sich aus diesen Beziehungen Verschiebungen von Verwandtschaftsbeziehungen auf Freundschafts-, Arbeits- und Nachbarschaftskontakte erkennen ließen. In seiner zweibändigen Studie „Townsmen or Tribesmen“ über die Xhosa in East London gelang Philip Mayer der

53 Vgl. dazu Coplan I994.

54 Redfield i94I.

55 Wirth I938.

56 Vgl auch Redfield i966.

57 Gluckman i961, 69.

58 Bott I957. 
Nachweis, dass die ungebildeten (Red) Xhosa ausschließlich Netzwerk-Beziehungen zu (Red) Xhosa sowohl in der Stadt wie auf dem Land unterhielten, während die gebildeten (School) Xhosa ein ländliches, verwandtschaftlich organisiertes Netzwerk sowie ein städtisches unterhielten, in dem sowohl (School) Xhosa als auch Mitglieder aus anderen Ethnien, wie Arbeits-, Sport- oder Gewerkschaftskameraden vertreten waren.59 Diese wichtigen Ergebnisse verdeutlichten, dass die Netzwerke Stadt und Land in unterschiedlichen Konstellationen miteinander verbanden. Die Realität des Dorfes wirkte über Normen, Verhaltensweisen, ökonomische und politische Bedingungen ebenso in die Städte hinein wie diese die Lebensweisen auf dem Dorf beeinflussten. ${ }^{60}$

Dieser grundlegende zyklische Zusammenhang zwischen Herkunft und Zielgebiet besteht auch in der gegenwärtigen Situation weiter, obwohl sich deren gesellschaftliche und politische Koordinaten weitgehend verändert haben. Handelte es sich bei der Migration der Wanderarbeiter - egal, ob im Copperbelt, in Südafrika oder von Burkina Faso in die Elfenbeinküste - noch um ein regionales Phänomen, so hat die Globalisierung zu einer transnationalen Erweiterung der Migrationsströme geführt. In diesem Zusammenhang sprechen Stephen Castles und Mark J. Miller von einem neuen Zeitalter der Migration. ${ }^{6}{ }$ Neu ist die quantitative Zunahme von internationalen Wanderungsbewegungen sowie die Umkehrungen ihrer Richtungen, da sich ehemalige Emigrations- zu Immigrationsländern transformierten. ${ }^{62}$ Dies gilt vornehmlich für Westeuropa, aber auch für viele andere Gebiete, da die Wanderungsströme nicht mehr uni-, sondern multidirektional ${ }^{63}$ verlaufen. Eine Folge dieser weltweiten Migration ist die Entwicklung von transnationalen Diaspora communities, ein Begriff, der in den letzten Jahren vielfache Deutungen und Umdeutungen erfahren hat. ${ }^{6}$ Ich benutze ihn hier zur Kennzeichnung ethnischer Gemeinschaften, deren Mitglieder entweder gewaltsam oder freiwillig ihre Herkunftsgesellschaften verlassen und sich über verschiedene Territorien verstreut haben. Trotz der verschiedenen Definitionen wird die Aufrechterhaltung grenzüberschreitender Netzwerkbeziehungen von den meisten Autoren als konstitutiv für eine Diaspora betrachtet. Roger Rouse spricht in diesem Zusammenhang von einem „transnational migrant circuit“, in dem Personen, Objekte, Ideen und virtuelle Welten zirkulieren.65 Interessant ist, dass den Objekten auch in den modernen Migrationsbewegungen eine wichtige Rolle zufällt. Sie werden häufig jenseits ihres unmittelbaren Gebrauchswertes als Zeichen kultureller Identität eingesetzt. Kleidung, Essen, Medikamente oder auch religiöse Artefakte symbolisieren kulturelle Werte

59 Mayer 1961.

6o Vgl. dazu Luig I996; Coplan I994.

6I Castles/Miller i993.

62 Im Folgenden lehne ich mich an meinen Aufsatz von 2007 an.

63 Damit ist auch schon eine weitere Veränderung innerhalb dieser Gesellschaften beschrieben: die Auflösung binärer Beziehungsmodelle - Nord-Süd, Peripherie-Zentrum - zugunsten einer Vielzahl und Vielfalt von Subzentren.

64 Vgl. Kokot 2002.

65 Rouse 1989. 
und markieren dadurch auch regionale oder ethnische Zugehörigkeiten, die aus „different regimes of value in space and time“ stammen, wie Arjun Appadurai in „The Social Life of Things" betont. 66

Damit sind wir bei einer weiteren - und wie ich glaube entscheidenden - Differenz im Migrationsverhalten angekommen. Die Verdichtung von Zeit und Raum, die Hartmut Rosa $^{67}$ in seinem kürzlich erschienenen Buch unter dem Begriff „Beschleunigung“ als Zeichen der Moderne zusammenfasst, verkürzt die Abstände wie auch die Inhalte zwischen Eigenem und Fremden. Dabei kommt den neuen Kommunikationstechnologien ohne Zweifel eine nicht zu überschätzende Bedeutung zu, wenn man bedenkt, dass Informationen wie Bilder in Sekundenschnelle von einem Land oder Kontinent zum anderen übermittelt werden können. Dies führt dazu, dass sich die so genannte Zwei-Welten-These, die mit Entfremdung und Entwurzelung einherging, in eine Welt des „in-between“ verwandelt oder in eine der „multiple roots“ auflöst.

\section{Schlussbetrachtung}

Ich habe in dieser Skizze einen kursorischen Überblick über verschiedene Formen von Mobilität gegeben, die in der ethnologischen Theoriebildung eine Rolle gespielt haben. Dabei war mir der Nachweis wichtig, dass das Nachdenken über Kultur und Gesellschaft in der deutschsprachigen Ethnologie immer auch mit dem Nachdenken über Ursprung und geschichtliche Entwicklung verbunden war. Die Prioritäten veränderten sich, indem die Diffusion der Objekte von der Sprache und diese wiederum von der Diffusion von Menschen abgelöst wurde. Die Migrationsforschung, die von der Chicago-Schule in den I92oer Jahren begründet wurde und über Redfield und die Manchester-Schule bei den heutigen Fragen globaler Migrationsströme angelangt ist, spiegelt eine deutliche Soziologisierung der Fragestellung wider. Fragen der Motivation, der Interaktion wie auch des Verlaufs der Migrationen werden im Zusammenhang mit sozialer Ungleichheit und Diskriminierung diskutiert. Im Unterschied zu den älteren Diskursen steht in den rezenten Studien eher das Individuum im Blickpunkt und seine Auseinandersetzung mit Fragen von Integration und Identität, die sich an Problemen von Ethnizität, Rasse und Klasse entzünden. Allerdings - und das scheint mir das grundlegend Neue zu sein - ist die Teilhabe der Migranten an ihrer Herkunftsgesellschaft nicht mehr an die (konkrete) Überwindung von Raum gebunden, sondern gestaltet sich zu einem virtuellen Hier- und Dortsein. Diese Erweiterung verschiebt dann aber auch die Frage von Identitäten, da sie vornehmlich an Kontexte, aber nicht mehr an Raumkonstrukte gebunden ist.

66 Appadurai i986, 4.

67 Rosa 2005. 


\section{Literatur}

Ackermann 1997

A. Ackermann, Ethnologische Migrationsforschung: ein Überblick. In: KEA, Zeitschrift für Kulturwissenschaften IO, I-28.

Appadurai I988 (zuerst 1986)

A. Appadurai, The Social Life of Things, Cambridge.

\section{Bormida 1967}

M. Bormida, Kultur und Kulturkreise - Ein Versuch zur theoretischen Ethnologie, In:

C.A. Schmitz (Hrsg.), Historische Völkerkunde, Frankfurt, I2I-I46.

\section{Bott 1957}

E. Bott, Family and Social Network, London.

\section{Brandstetter 2005}

A. Brandstetter, Erinnern und Trauern. Über Genozid-Gedenkstätten in Ruanda. In: W. Speitkamp (Hrsg.), Erinnerungsräume - Kommunikationsräume. Beiträge zur transkulturellen Begegnung in Afrika, München, 292-324.

\section{Castles/Miller 1993}

St. Castles/M.J. Miller, The Age of Migration. International Population Movements in the Modern World. London.

\section{Coplan 1994}

D.B. Coplan, In the Time of Cannibals. The Word Music of South Africa's Basotho Migrants. Chicago.

Curtin et al. 1978

P. Curtin/S. Feierman/L. Thompson/J. Vansina (Hrsg.), African History, London.

\section{Delafosse 1900}

M. Delafosse, Essai de la langue Agni, Paris.

\section{Eggert 1992}

M.K.H. Eggert, The Central African Rain Forest: Historical Speculation and Archaeological Facts. In: World Archaeology, Vol. 24, I. I-24.

\section{Fabian 200I}

J. Fabian, Im Tropenfieber. Wissenschaft und Wahn in der Erforschung Zentralafrikas, München.

\section{Gluckman I96I}

M. Gluckman, Anthropological Problems arising from the African Industrial Revolution. In: A. Southall (Hrsg.), Social Change in Modern Africa, London, 67-82.

\section{Graebner 1967}

F. Graebner, Kulturkreise und Kulturschichten. In: C.A. Schmitz (Hrsg.), Historische Völkerkunde, Frankfurt, 28-54. 


\section{Greenberg 1963}

J.H. Greenberg, The Languages of Africa. Den Haag/Bloomington.

Guthrie I967-7I

M. Guthrie, Comparative Bantu. An Introduction to the Comparative Linguistics and PreHistory of the Bantu-Language, 4 vol., Farnborough.

\section{Hannerz I980}

U. Hannerz, Exploring the City. Inquiries toward an Urban Anthropology, New York.

\section{Hart/Hart 1986}

T. B. Hart/J. A. Hart, The Ecological Basis of Hunter-Gatherer Subsistence in African Rain Forests: the Mbuti of Eastern Zaire. Human Ecology I4: 29-55.

\section{Heine 1973}

B. Heine, Zur genetischen Gliederung der Bantu-Sprachen. In: Afrika und Übersee ${ }_{5}^{6}$, I64-185.

\section{Heine et al. 1977}

B. Heine/H. Hof/R. Vossen, Neuere Ergebnisse zur Territorialgeschichte der Bantu (Juli I975), In: W. J. G. Möhlig/F. Rotland/B. Heine (Hrsg.), Zur Sprachgeschichte und Ethnohistorie in Afrika. Neue Beiträge afrikanistischer Forschungen, Berlin, 57-72.

\section{Hirschberg 1988}

W. Hirschberg, Neues Wörterbuch der Völkerkunde, Berlin

\section{Klein-Arendt 200I}

R. Klein-Arendt, Bantusprachen. In: J.E. Mabe (Hrsg.), Das Afrika Lexikon, Stuttgart, 70-72.

\section{Klute 1995}

G. Klute, Wo liegt Gottes Segen? Natur und Arbeit bei Nomaden der Sahara. In: U. Luig/ A. von Oppen (Hrsg.), Naturaneignung als symbolischer Prozess in afrikanischen Gesellschaften, 5I-64, Berlin, Forschungsschwerpunkt Moderner Orient, Arbeitshefte Nr. Io.

\section{Kokot 2002}

W. Kokot, Diaspora und transnationale Verflechtungen. In: B. Hauser-Schäublin/U. Braukämper (Hrsg.), Ethnologie der Globalisierung, Berlin, 95-IIO.

\section{Krüger 200I}

G. Krüger, Südafrika, Völker, Kulturen und Staaten, In: J. E. Mabe (Hrsg.), Das Afrika Lexikon, Stuttgart, 605-608.

\section{Lentz 2006}

C. Lentz, Ethnicity and the Making of History in Northern Ghana. Edinburgh.

\section{Luig 1980}

U. Luig, Konstitutionsbedingungen des Aschanti-Reiches: Zentralisierungsprozesse politischer Herrschaft: Von den Akan-Staaten zum Aschanti-Reich. In: R. Hanisch/R. Tetzlaff (Hrsg.), Historische Konstitutionsbedingungen des Staates in Entwicklungsländern, Frankfurt a. Main, II8-186. 


\section{Luig 1984}

U. Luig, Probleme bei der Erforschung oraler Traditionen. In: E. W. Müller/R. König/ K.P. Koepping/P. Drechsel (Hrsg.), Ethnologie als Sozialwissenschaft, Kölner Zeitschrift für Soziologie und Sozialpsychologie, Sonderheft 26, I78-I94.

\section{Luig 1985}

U. Luig, Orale Tradition als Interpretation von Geschichte. Die Sage von Aura Pokou. In: Anthropos 80. IOI-I24.

\section{Luig 1995}

U. Luig, Naturaneignung als symbolischer Prozess in afrikanischen Gesellschaften. In: U. Luig/A. von Oppen (Hrsg.), Naturaneignung als symbolischer Prozess in afrikanischen Gesellschaften, 29-50, Berlin, Forschungsschwerpunkt Moderner Orient, Arbeitshefte Nr. IO.

\section{Luig 1996}

U. Luig, Wanderarbeiter als Helden. Zwischen kolonialer Entfremdung und lokaler Selbstvergewisserung. In: Historische Anthropologie 3,4. 359-382.

\section{Luig 2007}

U. Luig, Diversity als Lebenszusammenhang - Ethnizität, Religion und Gesundheit im transnationalen Kontext. In: G. Krell/B. Riedmüller/B. Sieben/D. Vinz (Hrsg.), Diversity Studies. Grundlagen und disziplinäre Ansätze, Frankfurt/New York, 87-108.

\section{Luig/von Oppen 1995}

U. Luig/A. von Oppen, Einleitung: Zur Vergesellschaftung von Natur in Afrika, In: Naturaneignung als symbolischer Prozess in afrikanischen Gesellschaften, Berlin, 5-28, Forschungsschwerpunkt Moderner Orient, Arbeitshefte Nr. Io.

\section{Mayer 196I}

P. Mayer, Townsmen or Tribesmen. Cape Town.

\section{Meinhof 1938}

C. Meinhof, Die Entstehung der Bantu-Sprachen: In: Zeitschrift für Ethnologie 70, I44-I52.

\section{Möhlig I98I}

W. J. G. Möhlig, Stratification in the History of the Bantu Languages. In: Sprache und Geschichte in Afrika, Bd. 3, 25I-3I6.

\section{Mudimbe 1988}

Y.V. Mudimbe, The Invention of Africa. Gnosis, Philosophy and the Order of Knowledge. London.

\section{Mühlmann 1968}

W.E. Mühlmann, Geschichte der Anthropologie, Frankfurt.

\section{Neubert/Brandstetter 1996}

D. Neubert/A. Brandstetter, Historische und gesellschaftliche Hintergründe des Konflikts in Ruanda. In: P. Meyns (Hrsg.), Staat und Gesellschaft in Afrika, Erosions- und Reformprozesse, Hamburg, 409-424. 


\section{Obenga 1985}

T. Obenga, Les bantu. Langues, peuples, civilisations, Paris.

\section{Ogot 1968}

B.A. Ogot, The Role of the Pastoralist and Agriculturalist in African History. In: T. O. Ranger (Hrsg.), Emerging Themes on African History, Nairobi, I25-I33.

\section{Oliver 1966}

R. Oliver, The Problem of the Bantu-Expansion: In: Journal of African History, 7,3: 36I-373.

\section{Ratzel I89i}

F. Ratzel, Die afrikanischen Bögen. Ihre Verbreitung und Verwandtschaften. Leipzig,

\section{Redfield I94I}

R. Redfield, The Folk Culture of Yucatan. Chicago.

\section{Redfield I966}

R. Redfield, Die „Folk-Gesellschaft“. In: W. E. Mühlmann/E. W. Müller (Hrsg.), Kulturanthropologie, 327-355.

\section{Rosa 2005}

H. Rosa, Beschleunigung. Die Veränderung der Zeitstrukturen in der Moderne, Frankfurt a. Main.

\section{Rouse 1989}

R. Rouse, Mexican Migration to the United States, Family Relations in the Development of a Transnational Migrant Circuit. Univ. Dissertation, Stanford.

\section{Seligman 1930}

C. G. Seligman, Races of Africa. London.

\section{Sigrist 1968}

Ch. Sigrist, Regulierte Anarchie, Freiburg.

\section{Tempels 1945}

P. Tempels, La philosophie bantoue. Elisabethville.

\section{Turnbull 1983}

C.M. Turnbull, The Mbuti Pygmies. Change and Adaptation. New York.

\section{Vansina 1966}

J. Vansina, Kingdoms of the Savanna, Madison.

\section{Vansina 1973}

J. Vansina, Oral Tradition. A Study in Historical Methodology, Harmondsworth.

\section{Vansina 1974}

J. Vansina, Comment: Traditions of Genesis. In: Journal of African History I5,2. 317-322.

\section{Vansina 1985}

J. Vansina, Oral Tradition as History. London. 


\section{Vansina 1990}

J. Vansina, Paths in the Rainforests. Towards a History of Political Tradition in Equatorial Africa. London.

\section{Weiskel I980}

T. C. Weiskel, French Colonial Rule and the Baule Peoples. Resistance and Collaboration I889-I9II. New York.

\section{Wirth 1938}

L. Wirth, Urbanism as a Way of Life. In: American Journal of Sociology 44, I-24.

\section{Wirz 1997}

A. Wirz, Migrationen. Das Problem der Bantu-Expansion. In: J.G. Deutsch/A. Wirz (Hrsg.), Geschichte in Afrika, Einführung in Probleme und Debatten, 35-52, Berlin: Zentrum Moderner Orient, Studien 7.

\section{Wrigley 1960}

Ch. Wrigley, Speculations on the Prehistory of Africa. In: Journal of African History I. I89-203. 
